\title{
Strain-induced band gaps in bilayer graphene
}

\author{
B. Verberck, ${ }^{1,2}$ B. Partoens, ${ }^{1}$ F. M. Peeters, ${ }^{1}$ and B. Trauzettel ${ }^{2}$ \\ ${ }^{1}$ Departement Fysica, Universiteit Antwerpen, Groenenborgerlaan 171, B-2020 Antwerpen, Belgium \\ ${ }^{2}$ Institut für Theoretische Physik und Astrophysik, Universität Würzburg, Am Hubland, D-97070 Würzburg, Germany
}

(Received 17 November 2011; published 5 March 2012)

\begin{abstract}
We present a tight-binding investigation of strained bilayer graphene within linear elasticity theory, focusing on the different environments experienced by the $A$ and $B$ carbon atoms of the different sublattices. We find that the inequivalence of the $A$ and $B$ atoms is enhanced by the application of perpendicular strain $\varepsilon_{z z}$, which provides a physical mechanism for opening a band gap, most effectively obtained when pulling the two graphene layers apart. In addition, perpendicular strain introduces electron-hole asymmetry and can result in linear electronic dispersion near the $K$ point. Our findings suggest experimental means for strain-engineered band gaps in bilayer graphene.
\end{abstract}

DOI: 10.1103/PhysRevB.85.125403

PACS number(s): 77.65.Ly, 81.05.ue, 73.22.-f

\section{INTRODUCTION}

The synthesis of graphene-a single layer of carbon atoms arranged into a honeycomb structure-and measurements of its electronic properties in 2004 by Novoselov et al. ${ }^{1}$ has sparked off the development of a whole new research field, continuing to expand rapidly today in both experimental and theoretical directions. The high rate at which the graphene literature is growing is reflected by the regular appearance of reviews on graphene in recent years; for a selection of relevant accounts we refer to Refs. 2-8.

The original excitement about graphene not only came from its two dimensionality ${ }^{1,9}$ - flat two-dimensional (2D) crystals had not been successfully fabricated before and were even predicted to be unstable-but also from its electronic properties. ${ }^{1,9-11}$ Apart from novel fundamental physics, graphene boasts superior material properties, including high thermal conductivity, current density, carrier mobility, carrier mean free path, strongness, stiffness, elasticity, and impermeability.

Not only single graphene sheets but also stacks of a few graphene layers ${ }^{1}$ where the layers are coupled by van der Waals interactions as in graphite can be isolated. This has led to investigations of multilayer graphene, and in particular of bilayer graphene (Fig. 1, top). Interestingly, bilayer graphene displays (almost-)parabolic electronic dispersion at the $K$ points (Fig. 1, bottom left), making electrons behave differently $^{12-15}$ as compared to the single-layer case. Bilayer graphene offers the possibility of applying a bias voltage $W$ between the two layers, allowing to tune the band structure. In particular, the inequivalency of the two graphene layers then gives rise to a "Mexican-hat-like" band structure featuring a band gap ${ }^{15-19}$ of magnitude $E_{\mathrm{g}}=\frac{|e W| t_{\perp}}{\sqrt{(e W)^{2}+t_{\perp}^{2}}}$ (Fig. 1, bottom right), with $t_{\perp} \approx 0.377 \mathrm{eV}$ the interplane hopping parameter (see below). A tunable energy gap is important for possible electronic devices.

Another means of influencing (mono- or bilayer) graphene's electronic structure, currently receiving a lot of theoretical attention, ${ }^{20-28}$ is provided by mechanical deformations. It has, e.g., been shown that it is possible to conceive inhomogeneous strains in single-layer graphene such that they act as a high uniform magnetic field, therefore resulting in strain-induced Landau levels and a zero-field quantum Hall effect. ${ }^{20}$ In Ref. 21 , Pereira et al. elaborated a tight-binding description for uniaxially strained single-layer graphene and predicted that a band gap can form upon deformations-along preferred directions-beyond $20 \%$. Recent investigations on strained bilayer graphene include a standard tight-binding treatment of uniaxial strain, ${ }^{22}$ a description of elastic deformations and electron-phonon coupling in bilayer graphene by means of pseudomagnetic gauge fields, ${ }^{23}$ the effect of strain on the Landau-level spectrum and the quantum Hall effect, ${ }^{24,25}$ and the effect of strain in combination with an external electric field. ${ }^{26,27}$

In the present paper, we employ a nearest-neighbor tightbinding description and linear elasticity theory to show that a perpendicular strain component modifies the on-site energies of the two carbon sublattices in bilayer graphene, which can open a band gap at the $K$ point. The band gap is of a different nature than in the case of the Mexican-hat-like electronic dispersions. Uniform deformations parallel to the sheets are studied as well and are compared to the monolayer case. ${ }^{21}$

\section{STRAINED BILAYER-GRAPHENE}

We first consider the unstrained $A B$ (Bernal) stacking variant of bilayer graphene: two graphene layers (labeled 1 and 2) at $c=3.44 \AA$ apart with the $A$ atoms of layer 2 sitting directly on top of the $A$ atoms of layer 1 (Fig. 1, top). The $B$ atoms of layer 1 and the $B$ atoms of layer 2 have no direct neighbor in the opposite layer.

The band structure of bilayer graphene can be described within the tight-binding formalism. The nearest-neighbor tight-binding hamiltonian assumes one free $2 p_{z}$ electron provided by each carbon atom and reads

$$
\begin{aligned}
H= & V_{\mathrm{A}} \sum_{\alpha} \sum_{\vec{X}_{\alpha}} c_{\vec{X}_{\alpha}}^{\dagger} c_{\vec{X}_{\alpha}} \\
& +V_{\mathrm{B}}\left(\sum_{\vec{X}_{1}} c_{\vec{X}_{1}+\vec{d}_{1}}^{\dagger} c_{\vec{X}_{1}+\vec{d}_{1}}+\sum_{\vec{X}_{2}} c_{\vec{X}_{2}-\vec{d}_{1}}^{\dagger} c_{\vec{X}_{2}-\vec{d}_{1}}\right) \\
& -t\left(\sum_{\vec{X}_{1}} \sum_{l=1}^{3}\left[c_{\vec{X}_{1}}^{\dagger} c_{\vec{X}_{1}+\vec{d}_{l}}+c_{\vec{X}_{1}+\vec{d}_{l}}^{\dagger} c_{\vec{X}_{1}}\right]\right.
\end{aligned}
$$



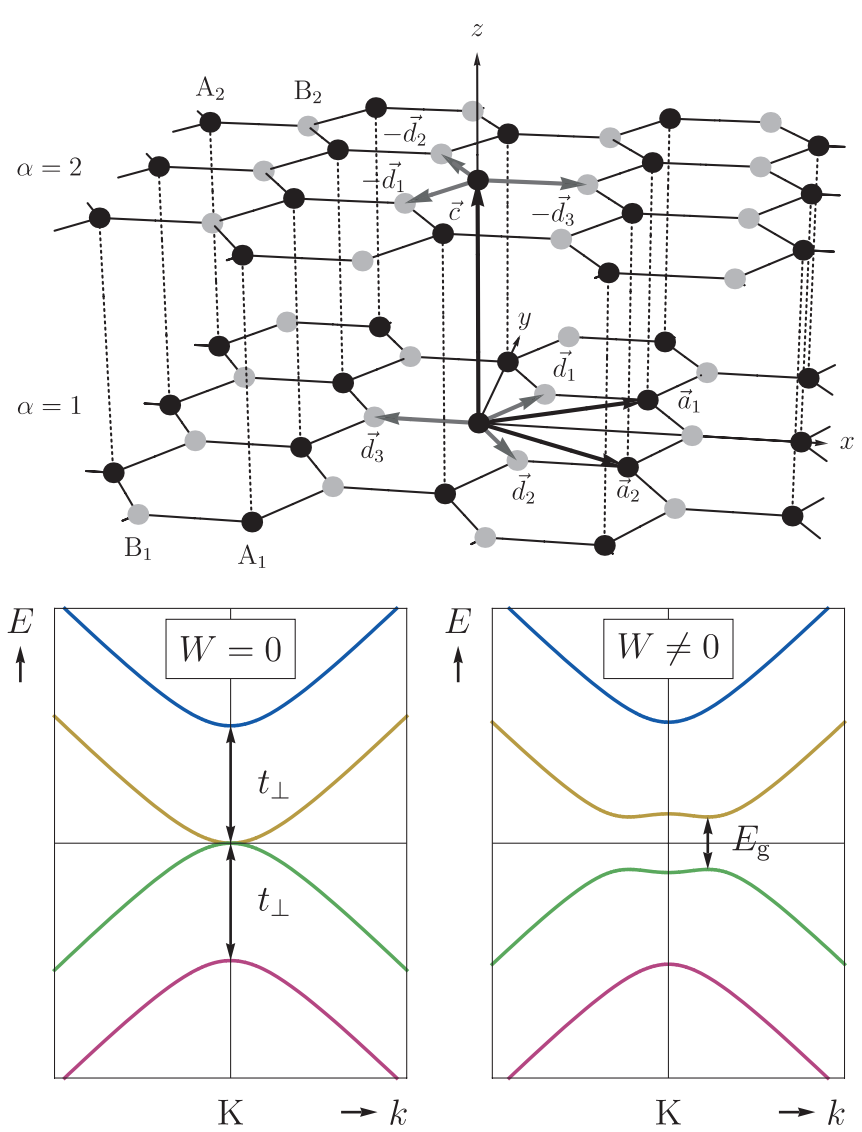

FIG. 1. (Color online) Graphene bilayer, with $A$ carbon atoms in black and $B$ carbon atoms in gray (top). Electronic spectrum near the $K$ point in absence (bottom left) and presence (bottom right) of a bias voltage $W$ between the layers.

$$
\begin{aligned}
& \left.+\sum_{\vec{X}_{2}} \sum_{l=1}^{3}\left[c_{\vec{X}_{2}}^{\dagger} c_{\vec{X}_{2}-\vec{d}_{l}}+c_{\vec{X}_{2}-\vec{d}_{l}}^{\dagger} c_{\vec{X}_{2}}\right]\right) \\
& +t_{\perp} \sum_{\vec{X}_{1}}\left[c_{\vec{X}_{1}}^{\dagger} c_{\vec{X}_{1}+\vec{c}}+c_{\vec{X}_{1}+\vec{c}}^{\dagger} c_{\vec{X}_{1}}\right] .
\end{aligned}
$$

The index $\alpha$ stands for the layer (1 or 2), the vectors $\vec{X}_{\alpha}$ are the lattice sites of the $A$ atoms of layer $\alpha$. Every $A$ atom in layer 1 is surrounded by three $B$ atoms at relative position vectors $\vec{d}_{1}=\frac{1}{2} a \vec{e}_{x}+\frac{\sqrt{3}}{2} a \vec{e}_{y}, \vec{d}_{2}=\frac{1}{2} a \vec{e}_{x}-\frac{\sqrt{3}}{2} a \vec{e}_{y}$, and $\vec{d}_{3}=$ $-a \vec{e}_{x}$, where the bond length $a$ has a value of $1.42 \AA$, and the $A$ atoms in layer 2 have neighboring $B$ atoms at relative positions $-\vec{d}_{l}, l=1,2,3$. The operators $c_{\vec{X}}^{\dagger}$ and $c_{\vec{X}}$ create and annihilate an electron at site $\vec{X}$, respectively. The vector $\vec{c}=c \vec{e}_{z}(c=3.35 \AA)$ connects two nearest-neighbor atoms in different graphene sheets. Values for the intra- and interplane hopping energies $t$ and $t_{\perp}$ are obtained from fitting the tight-binding model to experimental data for graphite. Here we use the values quoted in Ref. 29: $t=3.12 \mathrm{eV}$ and $t_{\perp}=0.377 \mathrm{eV}$. The on-site energies $V_{\mathrm{A}}$ and $V_{\mathrm{B}}$ differ slightly due to the different environments of $A$ and $B$ atoms. The difference $\Delta=\left|V_{\mathrm{A}}-V_{\mathrm{B}}\right| \approx 0.009 \mathrm{eV}$ (Ref. 29) is about two orders of magnitude smaller than the hopping parameter $t_{\perp}$ and is usually considered only in models going beyond the nearestneighbor tight-binding hamiltonian (1) where also $A_{1}-B_{2}$ and $B_{1}-B_{2}$ hoppings are taken into account (see, e.g., Ref. 14). We therefore put $V_{\mathrm{A}} \approx V_{\mathrm{B}} \equiv V$. The value of the on-site energies $V$ will turn out to be of critical importance when considering perpendicular strain; we will return to it later. For a comprehensive review on a complete tight-binding description of (unstrained) bilayer graphene, and in particular for a discussion of features in the electronic structure resulting from asymmetry of the diagonal (differences in on-site energies, $V_{\mathrm{A}} \neq V_{\mathrm{B}}$ ), we refer to Ref. 30 .

The direct-space hamiltonian (1) can be converted into a reciprocal-space hamiltonian by introducing four-component spinors:

$$
\begin{aligned}
& \Psi^{\dagger}(\vec{q})=\left(c_{\mathrm{A}_{1}}^{\dagger}(\vec{q}), \quad c_{\mathrm{B}_{1}}^{\dagger}(\vec{q}), \quad c_{\mathrm{A}_{2}}^{\dagger}(\vec{q}), \quad c_{\mathrm{B}_{2}}^{\dagger}(\vec{q})\right), \\
& \Psi(\vec{q})=\left(\begin{array}{c}
c_{\mathrm{A}_{1}}(\vec{q}) \\
c_{\mathrm{B}_{1}}(\vec{q}) \\
c_{\mathrm{A}_{2}}(\vec{q}) \\
c_{\mathrm{B}_{2}}(\vec{q})
\end{array}\right), \\
& H=\sum_{\vec{q}} \Psi^{\dagger}(\vec{q}) \mathfrak{H}(\vec{q}) \Psi(\vec{q}) \text {. }
\end{aligned}
$$

Here, the operators $c_{i}(\vec{q})\left(i=A_{1}, B_{1}, A_{2}, B_{2}\right)$ are the discrete (lattice) Fourier transforms of $c_{\vec{X}_{i}}$ :

$$
c_{i}(\vec{q})=\frac{1}{\sqrt{N}} \sum_{\vec{X}_{i}} c_{\vec{X}_{i}} e^{-i \vec{q} \cdot \vec{X}_{i}},
$$

where the $\vec{q}$ vectors of the first Brillouin zone are defined so that the properties $\sum_{\vec{q}} e^{i \vec{q} \cdot \vec{X}_{i}}=N \delta_{\vec{X}_{i}, \overrightarrow{0}}$ and $\sum_{\vec{X}_{i}} e^{i \vec{q} \cdot \vec{X}_{i}}=N \delta_{\vec{q}, \overrightarrow{0}}$ hold. The hamiltonian matrix $\mathfrak{H}(\vec{q})$ reads

$$
\mathfrak{H}(\vec{q})=\left(\begin{array}{cccc}
V & \zeta(\vec{q}) & t_{\perp} & 0 \\
\zeta(\vec{q})^{*} & V & 0 & 0 \\
t_{\perp} & 0 & V & \zeta(\vec{q})^{*} \\
0 & 0 & \zeta(\vec{q}) & V
\end{array}\right)
$$

with

$$
\zeta(\vec{q})=-t \sum_{l=1}^{3} e^{i \vec{q} \cdot \vec{d}_{l}}
$$

The band structure $\{E(\vec{q})\}$ is obtained by solving the secular equation $\operatorname{det}\left[\mathfrak{H}(\vec{q})-E(\vec{q}) I_{4}\right]=0$, with $I_{4}$ the $4 \times 4$ unit matrix, in the first Brillouin zone. At the $K$ and $K^{\prime}$ points, two electron energy bands touch each other at the Fermi level, making the material a semimetal (zero band gap).

Since the electronic properties of (bilayer) graphene are determined by the band structure near the $K^{\left({ }^{\prime}\right)}$ point $-\vec{q}_{\mathrm{K}}=$ $\frac{2 \pi}{3 a} \vec{e}_{x}+\frac{2 \pi}{3 \sqrt{3} a} \vec{e}_{y}$ and $\vec{q}_{\mathrm{K}^{\prime}}=\frac{2 \pi}{3 a} \vec{e}_{x}-\frac{2 \pi}{3 \sqrt{3} a} \vec{e}_{y}$-it is convenient to make a Taylor expansion of $\mathfrak{H}(\vec{q})$ around $\vec{q}_{\mathrm{K}^{\left({ }^{\prime}\right)}}$. With $\vec{q}=\vec{q}_{\mathrm{K}}+$ $\vec{k}$ and retaining only lowest-order terms in $k$, the hamiltonian 
matrix near $\vec{q}_{\mathrm{K}}$ becomes

$$
\mathfrak{H}^{\mathrm{K}}(\vec{k})=\left(\begin{array}{cccc}
V & \frac{3 a t}{2} k e^{i \phi} e^{-i(\pi / 6)} & t_{\perp} & 0 \\
\frac{3 a t}{2} k e^{-i \phi} e^{i(\pi / 6)} & V & 0 & 0 \\
t_{\perp} & 0 & V & \frac{3 a t}{2} k e^{-i \phi} e^{i(\pi / 6)} \\
0 & 0 & \frac{3 a t}{2} k e^{i \phi} e^{-i(\pi / 6)} & V
\end{array}\right) .
$$

Here, $\phi$ is defined via $k_{x}+i k_{y}=k e^{i \phi}$. For $\vec{q}=\vec{q}_{\mathrm{K}^{\prime}}+\vec{k}$, the hamiltonian matrix $\mathfrak{H}^{\mathrm{K}^{\prime}}(\vec{k})$ is the complex conjugate of $\mathfrak{H}^{\mathrm{K}}(\vec{k})$. Note that the quantity $\frac{3 a t}{2}$ can be rewritten as $\hbar v_{\mathrm{F}}$, with $v_{\mathrm{F}}$ the Fermi velocity. Diagonalization of Eq. (6) results in the dispersion shown in the left bottom of Fig. 1.

As mentioned in the Introduction, the application of a bias voltage $W$ between layers 1 and 2 [replacing the elements $\mathfrak{H}_{11}^{\mathrm{K}^{(\prime)}}(\vec{k})$ and $\mathfrak{H}_{22}^{\mathrm{K}^{(\prime)}}(\vec{k})$ by $e W / 2$ and $\mathfrak{H}_{33}^{\mathrm{K}^{\left(^{\prime}\right)}}(\vec{k})$ and $\mathfrak{H}_{44}^{\mathrm{K}^{\left({ }^{\prime}\right)}}(\vec{k})$ by $\left.-e W / 2\right]$ results in a finite band gap. ${ }^{15-19}$ However, this band gap lies not at $\vec{q}=\vec{q}_{\mathrm{K}^{(\prime)}}$ but at a $q$ vector slightly away from $q_{\mathrm{K}^{(\prime)}}$ (Fig. 1, bottom right). In the following, we will show that applying a strain to the bilayer graphene lattice can also lead to a band gap.

In the presence of a displacement field $\vec{u}(\vec{X})$, the position of an atom formerly at $\vec{X}$ is $\vec{X}+\vec{u}(\vec{X})$. The effect of a small deformation of the lattice can be written as a correction $\delta H$ to the original hamiltonian $H$, with

$$
\begin{aligned}
\delta H= & \delta V_{\mathrm{A}} \sum_{\alpha} \sum_{\vec{X}_{\alpha}} c_{\vec{X}_{\alpha}}^{\dagger} c_{\vec{X}_{\alpha}}+\delta V_{\mathrm{B}}\left(\sum_{\vec{X}_{1}} c_{\vec{X}_{1}+\vec{d}_{1}}^{\dagger} c_{\vec{X}_{1}+\vec{d}_{1}}+\sum_{\vec{X}_{2}} c_{\vec{X}_{2}-\vec{d}_{1}}^{\dagger} c_{\vec{X}_{2}-\vec{d}_{1}}\right) \\
& -\sum_{\vec{X}_{1}} \sum_{l=1}^{3} \delta t_{l}\left[c_{\vec{X}_{1}}^{\dagger} c_{\vec{X}_{1}+\vec{d}_{l}}+c_{\vec{X}_{1}+\vec{d}_{l}}^{\dagger} c_{\vec{X}_{1}}\right]-\sum_{\vec{X}_{2}} \sum_{l=1}^{3} \delta t_{l}\left[c_{\vec{X}_{2}}^{\dagger} c_{\vec{X}_{2}-\vec{d}_{l}}+c_{\vec{X}_{2}-\vec{d}_{l}}^{\dagger} c_{\vec{X}_{2}}\right]+\sum_{\vec{X}_{1}} \delta t_{\perp}\left[c_{\vec{X}_{1}}^{\dagger} c_{\vec{X}_{1}+\vec{d}}+c_{\vec{X}_{1}+\vec{d}}^{\dagger} c_{\vec{X}_{1}}\right] .
\end{aligned}
$$

Within the nearest-neighbor tight-binding approximation, the changes in on-site and hopping energy parameters $V_{\mathrm{A}}, V_{\mathrm{B}}, t$, and $t_{\perp}$ are related to changes in nearest-neighbor interatomic distances (bond lengths). We stress that it is therefore important to distinguish between $A$ and $B$ sites since they have different environments in the bilayer. As pointed out before, an $A$ site has three in-plane nearest-neighbor $B$ sites and one neighboring $A$ site in the opposite layer at a distance $c+\delta c$; a $B$ site has only the three surrounding in-plane $A$ sites as nearest neighbors (see Fig. 1, top). Denoting the three (not necessarily equal) changed bond lengths between neighboring in-plane $A$ and $B$ atoms by $a_{l}=a+\delta a_{l}(l=1,2,3)$, we have for the corrections to the on-site energies to linear order in the deformations

$$
\begin{aligned}
& \delta V_{\mathrm{A}}=\left.\sum_{l=1}^{3} \frac{\partial V}{\partial a}\right|_{a} \delta a_{l}+\left.\frac{\partial V}{\partial c}\right|_{c} \delta c, \\
& \delta V_{\mathrm{B}}=\left.\sum_{l=1}^{3} \frac{\partial V}{\partial a}\right|_{a} \delta a_{l}
\end{aligned}
$$

for each of the two layers. For the hopping parameters we have

$$
\begin{aligned}
\delta t_{l} & =\left.\frac{\partial t}{\partial a}\right|_{a} \delta a_{l}, \\
\delta t_{\perp} & =\left.\frac{\partial t_{\perp}}{\partial c}\right|_{c} \delta c .
\end{aligned}
$$

In the following we will drop the attributes $\left.\right|_{a}$ and $\left.\right|_{c}$. The link between the corrections $\delta V_{\mathrm{A}}, \delta V_{\mathrm{B}}, \delta t_{l}$, and $\delta t_{\perp}$ and the displacement field $\vec{u}(\vec{X})$ then comes from considering the bond length corrections $\delta a$ and $\delta c$. Details of the calculations and approximations involved are given in the Appendix; the resulting correction $\delta \mathfrak{H}(\vec{q})$ to the hamiltonian matrix $\mathfrak{H}(\vec{q})$ reads

$$
\delta \mathfrak{H}(\vec{q})=\left(\begin{array}{cccc}
\frac{3 a}{2} \frac{\partial V}{\partial a}\left(\varepsilon_{x x}+\varepsilon_{y y}\right)+c \frac{\partial V}{\partial c} \varepsilon_{z z} & \delta \zeta(\vec{q}) & c \frac{\partial t_{\perp}}{\partial c} \varepsilon_{z z} & 0 \\
\delta \zeta(\vec{q})^{*} & \frac{3 a}{2} \frac{\partial V}{\partial a}\left(\varepsilon_{x x}+\varepsilon_{y y}\right) & 0 & 0 \\
c \frac{\partial t_{\perp}}{\partial c} \varepsilon_{z z} & 0 & \frac{3 a}{2} \frac{\partial V}{\partial a}\left(\varepsilon_{x x}+\varepsilon_{y y}\right)+c \frac{\partial V}{\partial c} \varepsilon_{z z} & \delta \zeta(\vec{q})^{*} \\
0 & 0 & \delta \zeta(\vec{q}) & \frac{3 a}{2} \frac{\partial V}{\partial a}\left(\varepsilon_{x x}+\varepsilon_{y y}\right)
\end{array}\right)
$$


with

$$
\begin{aligned}
\delta \zeta(\vec{q}) & =-\sum_{l=1}^{3} \delta t_{l} e^{i \vec{q} \cdot \vec{d}_{l}} \\
& =-a \frac{\partial t}{\partial a} e^{i(1 / 2) a q_{x}} e^{i(\pi / 6)}\left\{\left[\frac{1}{2} \cos \left(\frac{\sqrt{3}}{2} a q_{y}\right)+e^{-i(3 / 2) a q_{x}}\right] \varepsilon_{x x}+\frac{3}{2} \cos \left(\frac{\sqrt{3}}{2} a q_{y}\right) \varepsilon_{y y}+\sqrt{3} i \sin \left(\frac{\sqrt{3}}{2} a q_{y}\right) \varepsilon_{x y}\right\} .
\end{aligned}
$$

Here, the quantities $\varepsilon_{i j}(i, j=x, y, z)$ are elements of the strain tensor $[\varepsilon]$, the general definition of which involves derivatives of the displacement field components to the coordinates. For the uniform displacements we mostly consider in this work the elements $\varepsilon_{i j}$ can be related to relative increments/decrements of the bond lengths occurring in the lattice (see the Appendix): $\vec{u}(\vec{X})=[\varepsilon] \vec{X}$.

The leading correction $\delta \mathfrak{H}^{\mathrm{K}}$ to the hamiltonian matrix $\mathfrak{H}^{\mathrm{K}}(\vec{k})$ at the $K$ point is $k$ independent and reads

$$
\begin{aligned}
& \delta \mathfrak{H}^{\mathrm{K}} \\
& =\left(\begin{array}{cccc}
\frac{3 a}{2} \frac{\partial V}{\partial a}\left(\varepsilon_{x x}+\varepsilon_{y y}\right)+c \frac{\partial V}{\partial c} \varepsilon_{z z} & -i \frac{3 a}{4} \frac{\partial t}{\partial a}\left(-\varepsilon_{x x}+\varepsilon_{y y}+2 i \varepsilon_{x y}\right) & c \frac{\partial t_{\perp}}{\partial c} \varepsilon_{z z} & 0 \\
i \frac{3 a}{4} \frac{\partial t}{\partial a}\left(-\varepsilon_{x x}+\varepsilon_{y y}-2 i \varepsilon_{x y}\right) & \frac{3 a}{2} \frac{\partial V}{\partial a}\left(\varepsilon_{x x}+\varepsilon_{y y}\right) & 0 & 0 \\
c \frac{\partial t_{\perp}}{\partial c} \varepsilon_{z z} & 0 & \frac{3 a}{2} \frac{\partial V}{\partial a}\left(\varepsilon_{x x}+\varepsilon_{y y}\right)+c \frac{\partial V}{\partial c} \varepsilon_{z z} & i \frac{3 a}{4} \frac{\partial t}{\partial a}\left(-\varepsilon_{x x}+\varepsilon_{y y}-2 i \varepsilon_{x y}\right) \\
0 & 0 & -i \frac{3 a}{4} \frac{\partial t}{\partial a}\left(-\varepsilon_{x x}+\varepsilon_{y y}+2 i \varepsilon_{x y}\right) & \frac{3 a}{2} \frac{\partial V}{\partial a}\left(\varepsilon_{x x}+\varepsilon_{y y}\right)
\end{array}\right) .
\end{aligned}
$$

For $\vec{q}=\vec{q}_{\mathrm{K}^{\prime}}+\vec{k}$, the hamiltonian matrix correction $\delta \mathfrak{H}^{\mathrm{K}^{\prime}}$ is the complex conjugate of $\delta \mathfrak{H}^{\mathrm{K}}$. The $K$-point hamiltonian correction for a strained single graphene layer, of the form

$$
\left(\begin{array}{cc}
g_{1}\left(\varepsilon_{x x}+\varepsilon_{y y}\right) & g_{2}\left(\varepsilon_{x x}-\varepsilon_{y y}+2 i \varepsilon_{x y}\right) \\
g_{2}^{*}\left(\varepsilon_{x x}-\varepsilon_{y y}-2 i \varepsilon_{x y}\right) & g_{1}\left(\varepsilon_{x x}+\varepsilon_{y y}\right)
\end{array}\right),
$$

has been derived before in the context of electron-phonon coupling in carbon nanotubes, ${ }^{31}$ where the term proportional to $g_{1}$ is a deformation potential, a concept going back to Bardeen and Shockley, ${ }^{32}$ and the term proportional to $g_{2}$ corresponds to a bond-length change. However, to our knowledge, the derivation of the strained bilayer hamiltonian [Eqs. (10) and (11)] as given in the Appendix-with particular emphasis on the asymmetry on the diagonal-has not been reported before.

\section{PERPENDICULAR UNIFORM STRAIN}

We now consider the bilayer-specific possibility of perpendicular strain, $\varepsilon_{z z}$, associated with a change from the interlayer distance $c$ to $c^{\prime}=c\left(1+\varepsilon_{z z}\right)$. Putting the in-plane strain tensor elements to zero, the hamiltonian $\mathfrak{H}^{\mathrm{K}}(\vec{k})+\delta \mathfrak{H}^{\mathrm{K}}$ takes on the form

$$
\begin{aligned}
\mathfrak{H}^{\mathrm{K}}(\vec{k})+\delta \mathfrak{H}^{\mathrm{K}} & \\
\quad= & \left(\begin{array}{cccc}
V+c \frac{\partial V}{\partial c} \varepsilon_{z z} & \hbar v_{\mathrm{F}} k e^{i \phi} & t_{\perp}+c \frac{\partial t_{\perp}}{\partial c} \varepsilon_{z z} & 0 \\
\hbar v_{\mathrm{F}} k e^{-i \phi} & V & 0 & 0 \\
t_{\perp}+c \frac{\partial t_{\perp}}{\partial c} \varepsilon_{z z} & 0 & V+c \frac{\partial V}{\partial c} \varepsilon_{z z} & \hbar v_{\mathrm{F}} k e^{-i \phi} \\
0 & 0 & \hbar v_{\mathrm{F}} k e^{i \phi} & V
\end{array}\right) .
\end{aligned}
$$

The phase factors $e^{\mp i(\pi / 6)}$ present in Eq. (6) have been eliminated by means of a redefinition of the operators for $B$ sites [Eqs. (A24a) and (A24b)].

The symmetry breaking along the diagonal exhibited by the hamiltionian matrix (14) is unusual since it distinguishes not between layers (as, e.g., a bias voltage between the two layers would do) but between $A$ and $B$ sublattices. Interestingly, the hamiltonian (14) displays the possibility of the opening of a band gap at $k=0$ : if

$$
\left|c \frac{\partial V}{\partial c} \varepsilon_{z z}\right|>t_{\perp}+c \frac{\partial t_{\perp}}{\partial c} \varepsilon_{z z},
$$

the degeneracy of the bands at $k=0$ is lifted, as illustrated in Fig. 2. [Criterion (15), an analytical result, holds when $t_{\perp}+c \frac{\partial t_{\perp}}{\partial c} \varepsilon_{z z}>0$, i.e., when $c\left|\frac{\partial t_{\perp}}{\partial c} \varepsilon_{z z}\right|$ is small compared to $t_{\perp}$.] Recently, Mucha-Kruczyński et al. ${ }^{30}$ examined the asymmetry of the (unstrained) graphene bilayer hamiltonian's diagonal. The possibility of band-gap openings and electron-hole asymmetry, as encountered here in Fig. 2, due to different on-site energies, was realized. Here, we show that strain enhances the diagonal's asymmetry [Eq. (10)] and that elastic deformations therefore provide a physical mechanism for the band-structure modifications discussed in Ref. 30.

To check whether the possibility of a strain-induced band gap is experimentally relevant, a more quantitative investigation of criterion (15) is in order. First, the variation of the hopping parameter $t_{\perp}$ with interlayer distance $c$ can be estimated using Harrison's relation

$$
\frac{\partial t_{\perp}}{\partial c} \approx-2 \frac{t_{\perp}}{c},
$$

which follows from an assumed inverse-square dependence of $t_{\perp}$ on $c .{ }^{33}$ The inequality (15) then becomes

$$
c\left|\frac{\partial V}{\partial c}\right|\left|\varepsilon_{z z}\right| \gtrsim t_{\perp}\left(1-2 \varepsilon_{z z}\right) .
$$

For expansion $\left(\varepsilon_{z z}>0\right)$, this can be rewritten as

$$
c\left|\frac{\partial V}{\partial c}\right| \gtrsim t_{\perp}\left(\frac{1}{\varepsilon_{z z}}-2\right),
$$



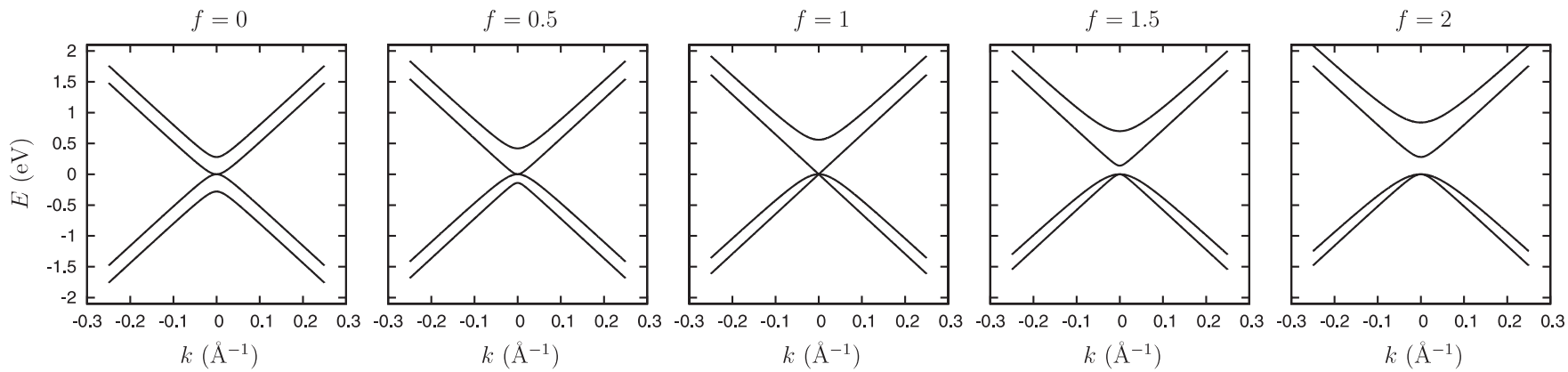

FIG. 2. Low- $k$ band structure $E(k)$ for perpendicularly uniformly strained bilayer graphene for various values of $\left|c \frac{\partial V}{\partial c} \varepsilon_{z z}\right|=f\left(t_{\perp}+c \frac{\partial t_{\perp}}{\partial c} \varepsilon_{z z}\right)$.

while for contraction $\left(\varepsilon_{z z}<0\right)$, one obtains

$$
c\left|\frac{\partial V}{\partial c}\right| \gtrsim t_{\perp}\left(-\frac{1}{\varepsilon_{z z}}+2\right) .
$$

The intra- and interplane hopping parameters have values of $t=3.12 \mathrm{eV}$ and $t_{\perp}=0.377 \mathrm{eV}$, respectively. The interplane distance is $c=3.35 \AA$, so that $\frac{t_{\perp}}{c}=0.113 \mathrm{eV} / \AA$. Using these values, we have plotted the quantities $\frac{t_{\perp}}{c}\left(\frac{1}{\varepsilon_{z z}}-2\right)$ and $\frac{t_{\perp}}{c}\left(\frac{1}{\left|\varepsilon_{z z}\right|}+2\right)$ in Fig. 3. Interestingly, it follows that the band-gap formation criterion is reached more easily (smaller $\left.\left|\varepsilon_{z z}\right|\right)$ in the case of expansion.

To obtain a physically meaningful estimate for the quantity $\left|\frac{\partial V}{\partial c}\right|$ we proceed as follows. First, we recall the tight-binding definition of $V$ :

$$
V \equiv \varepsilon_{2 p_{z}}=\int d \vec{r} \psi^{*}(\vec{r}) U(\vec{r}) \psi(\vec{r})
$$

Here, $\psi(\vec{r})$ is the $2 p_{z}$ electron wave function for a carbon atom, and $U(\vec{r})$ is the periodic potential of the lattice. Surprisingly, while the tight-binding formalism is a standard method for modeling band structures - especially for carbon structures-a numerical value for $V \equiv \varepsilon_{2 p_{z}}$ has not yet been established in the literature. The plausible reason is that when the difference between $V_{\mathrm{A}}$ and $V_{\mathrm{B}}$ is neglected (see Sec. II), the presence

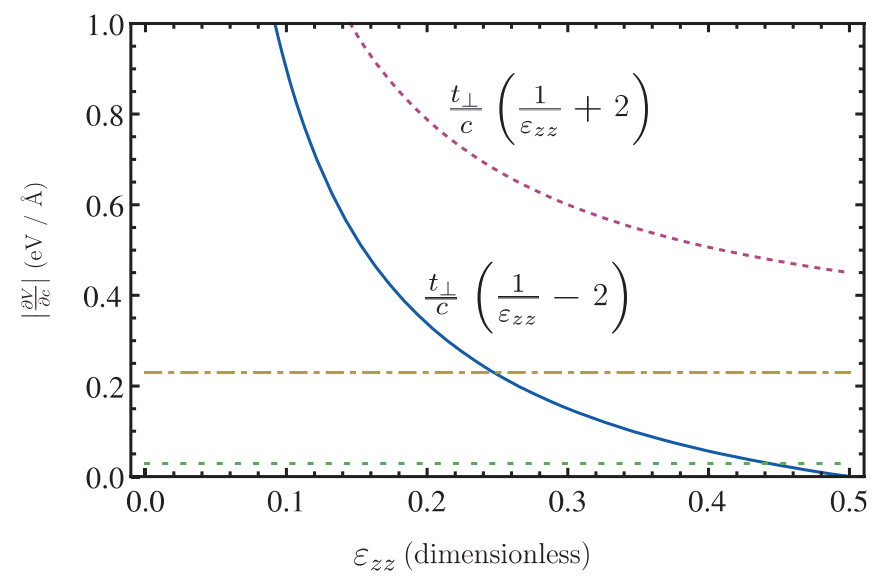

FIG. 3. (Color online) Plots of the variation of $\frac{t_{\perp}}{c}\left(\frac{1}{\varepsilon_{z z}}-2\right)$ (blue, full line, expansion) and $\frac{t_{\perp}}{c}\left(\frac{1}{\varepsilon_{z z}}+2\right)$ (violet, dashed line, contraction) with $\varepsilon_{z z}$. The two horizontal lines represent the values of $\left|\frac{\partial V}{\partial c}\right|$ resulting from using the two extreme values for $V$ considered in the present work- $V=-2 \mathrm{eV}$ (olive, dashed-dotted line) and $V=-0.25 \mathrm{eV}$ (green, dashed line). of $V$ on the diagonal of the hamiltonian matrix merely results in an overall shift of the energy bands, making its actual value redundant. In Ref. 34, Reich et al. present a detailed comparison of tight-binding and $a b$ initio energy dispersion calculations for graphene and provide fits of the tight-binding parameters to the band structures obtained by density-functional theory. Two variants were considered: one fitting the full $M \Gamma K M$ path in $k$ space and one where only $\vec{k}$ vectors yielding optical transitions with energies less than $4 \mathrm{eV}$ were taken into account, resulting in $\varepsilon_{2 p_{z}}=-0.28$ and $-2.03 \mathrm{eV}$, respectively. We will therefore consider the range $-0.25 \mathrm{eV} \geqslant \varepsilon_{2 p_{z}} \geqslant-2 \mathrm{eV}$. The simplest way of modeling $U(\vec{r})$ is to put positively charged ions (charge $1 e>0$ ) at $\vec{r}=\overrightarrow{0}$ and $\vec{r}=c^{\prime} \vec{e}_{z}$ :

$U(\vec{r}) \propto-\frac{1}{|\vec{r}|}-\frac{1}{\left|\vec{r}-c^{\prime} \vec{e}_{z}\right|}=-\frac{1}{r}-\frac{1}{\sqrt{r^{2}-2 c^{\prime} r \cos \theta+c^{\prime 2}}}$,

where spherical coordinates have been introduced. For the carbon $2 p_{z}$ electron wave function, we follow the common practice of taking the hydrogenlike $2 p_{z}$ orbital:

$$
\psi(\vec{r}) \propto r e^{-r / 2 a_{0}} \cos \theta,
$$

where $a_{0}=0.529 \AA$ is the Bohr radius. We then get for $V$ the expression

$$
\begin{aligned}
V= & C \int_{0}^{\infty} r^{2} d r \int_{0}^{\pi} \sin \theta d \theta r^{2} e^{-r / a_{0}} \cos ^{2} \theta \\
& \times\left(-\frac{1}{r}-\frac{1}{\sqrt{r^{2}-2 c^{\prime} r \cos \theta+c^{\prime 2}}}\right),
\end{aligned}
$$

where the proportionality factors left unspecified in Eqs. (21) and (22), together with the factor $2 \pi$ coming from the azimuthal integration, have been collected into the factor $C$. The integrals

$$
S_{n c^{\prime}}(r)=\int_{0}^{\pi} d \theta \frac{\sin \theta \cos ^{2} \theta}{\sqrt{r^{2}-2 n c^{\prime} r \cos \theta+\left(n c^{\prime}\right)^{2}}}
$$

entering Eq. (23) can be solved analytically:

$$
\begin{aligned}
S_{0}(r) & =\frac{2}{3 r}, \\
S_{n c^{\prime}>0}(r) & =\left\{\begin{array}{l}
\frac{2}{15} \frac{5\left(n c^{\prime}\right)^{2}+2 r^{2}}{\left(n c^{\prime}\right)^{3}} \text { if } 0 \leqslant r \leqslant n c^{\prime} \\
\frac{2}{15} \frac{2\left(n c^{\prime}\right)^{2}+5 r^{2}}{r^{3}} \text { if } r>n c^{\prime}
\end{array} .\right.
\end{aligned}
$$




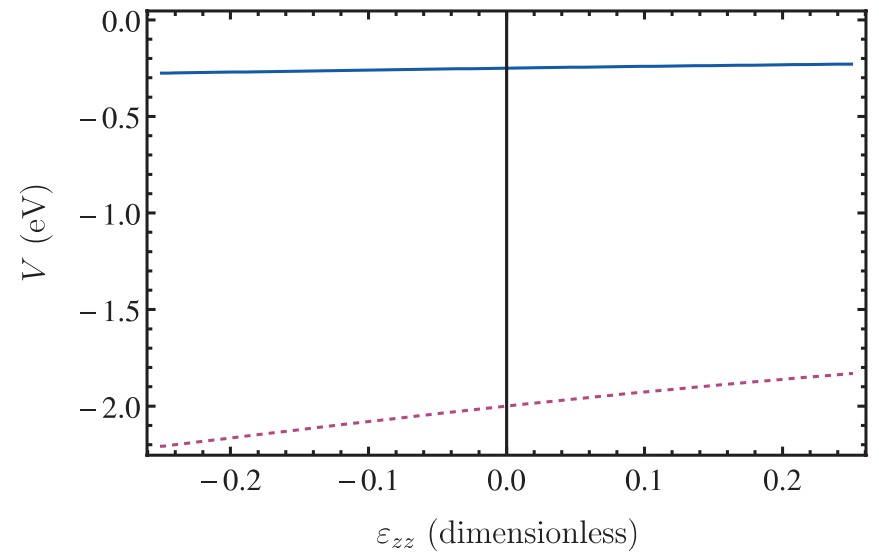

FIG. 4. (Color online) Plots of the variation of $V\left(c^{\prime}\right)$ with $c^{\prime}=$ $c\left(1+\varepsilon_{z z}\right)$ for $V(c)=-0.25 \mathrm{eV}$ (blue, full line) and $V(c)=-2 \mathrm{eV}$ (violet, dashed line).

For $c^{\prime}=c=3.35 \AA$, the integral

$$
D(c)=\int_{0}^{\infty} d r r^{4} e^{-r / a_{0}}\left[S_{0}(r)+S_{1 c}(r)\right]
$$

has the value $D(c)=0.539 \AA^{4}$. From $V=C D(c)$ we can then obtain the "calibration value" $C=V / D(c)$. For the values $V=-0.25 \mathrm{eV}$ and $V=-2 \mathrm{eV}$, we obtain $C=$ $-0.463 \mathrm{eV} / \AA^{-4}$ and $C=-3.709 \mathrm{eV} / \AA^{-4}$, respectively. The dependence of $V$ on $c^{\prime}$ reads $V\left(c^{\prime}\right)=C D\left(c^{\prime}\right)$; it is visualized in Fig. 4. Clearly, a linear approximation in the range $-0.25 \leqslant$ $\varepsilon_{z z} \leqslant 0.25$ is justifiable, and the value of $\frac{\partial V}{\partial c}$ can be easily determined numerically. We find $\frac{\partial V}{\partial c}=0.0287 \mathrm{eV} / \AA$ and $0.230 \mathrm{eV} / \AA ̊$ for $V=-0.25 \mathrm{eV}$ and $V=-2 \mathrm{eV}$, respectively; these values are marked by horizontal lines in Fig. 3 .

$$
\mathfrak{H}(\vec{k})=\left(\begin{array}{cc}
V+\frac{3 a}{2} \frac{\partial V}{\partial a}\left(\varepsilon_{x x}+\varepsilon_{y y}\right) & \zeta(\vec{q})+\delta \zeta(\vec{q}) \\
\zeta^{*}(\vec{q})+\delta \zeta^{*}(\vec{q}) & V+\frac{3 a}{2} \frac{\partial V}{\partial a}\left(\varepsilon_{x x}+\varepsilon_{y y}\right) \\
t_{\perp} & 0 \\
0 & 0
\end{array}\right.
$$

The four solutions of the corresponding secular equation are

$$
\begin{aligned}
& E_{ \pm, \pm}(\vec{q}) \\
& = \pm \sqrt{\frac{t_{\perp}^{2}+2|\zeta(\vec{q})+\delta \zeta(\vec{q})|^{2} \pm t_{\perp} \sqrt{t_{\perp}^{2}+4|\zeta(\vec{q})+\delta \zeta(\vec{q})|^{2}}}{2}} .
\end{aligned}
$$

There can only be a band gap when $\zeta(\vec{q})+\delta \zeta(\vec{q})$ differs from zero for all $\vec{q}$ vectors. The same condition arises from the monolayer strain problem, where the two solutions of the secular equation $\operatorname{read} E_{ \pm}(\vec{q})= \pm|\zeta(\vec{q})+\delta \zeta(\vec{q})|$ (leading to the Dirac cone at the $K^{\prime}$ point for $\delta \zeta(\vec{q})=0$ ). Pereira et al. ${ }^{21}$ have investigated the behavior of $|\zeta(\vec{q})+\delta \zeta(\vec{q})|$ in detail. Using the isotropy of a $2 \mathrm{D}$ hexagonal lattice, the strain tensor
Within the present model, it follows that in the case of a large tight-binding on-site energy parameter $(|V|=2 \mathrm{eV})$, a band gap opens for positive strains larger than $\varepsilon_{z z} \approx 0.25$ (see Fig. 3), corresponding to interlayer distances larger than $c^{\prime} \approx 4.19 \AA$. For $\varepsilon_{z z} \approx 30 \%$, the band gap's magnitude is about $125 \mathrm{meV}$ (Fig. 2, $f=1.5$ ).

We recall that formally, criterion (15) is valid when the right-hand side is positive, i.e., when $\varepsilon_{z z}<0.5$, hence the upper limit of $\varepsilon_{z z}=0.5$ in Fig. 3. Going beyond strains of $50 \%$ (both positive and negative) would be well outside the validity of the assumption of small displacements $\vec{u}(\vec{X})$, on which the hamiltonian matrix (10) relies (see the Appendix). For $\varepsilon \sim 0.25$, neglected contributions are of the order $\varepsilon^{2} \sim$ 0.063 , which is still acceptable. Similarly, we point out that the unphysical behavior of an ever-increasing band gap with increasing $\varepsilon_{z z}$ must become invalid when linear elasticity, i.e., the assumption of small bond length changes [Eq. (A2)], fails.

Based on the foregoing elaborations, stating that the opening of a strain-induced band gap by pulling apart the two graphene layers may be experimentally observed is a fair conclusion. Our main purpose here is not to provide accurate predictions but rather to point out the consequences of the symmetry breaking along the diagonal in the hamiltonian of strained bilayer graphene. Accurate density-functional theory calculations could provide better numerical estimates, but most importantly, experiments should be undertaken to investigate the effect on the band structure upon pushing together or pulling apart the graphene layers.

\section{SYMMETRIC UNIFORM $x y$ STRAIN}

We next consider pulling or pushing the bilayer in a direction parallel to the graphene sheets $\left(\varepsilon_{z z}=0\right)$. The tightbinding hamiltonian then reads

$$
\left.\begin{array}{cc}
t_{\perp} & 0 \\
0 & 0 \\
V+\frac{3 a}{2} \frac{\partial V}{\partial a}\left(\varepsilon_{x x}+\varepsilon_{y y}\right) & \zeta^{*}(\vec{q})+\delta \zeta^{*}(\vec{q}) \\
\zeta(\vec{q})+\delta \zeta(\vec{q}) & V+\frac{3 a}{2} \frac{\partial V}{\partial a}\left(\varepsilon_{x x}+\varepsilon_{y y}\right)
\end{array}\right) .
$$

can be written as

$$
[\varepsilon]=\varepsilon\left(\begin{array}{cc}
\cos ^{2} \theta-\sigma \sin ^{2} \theta & (1+\sigma) \cos \theta \sin \theta \\
(1+\sigma) \cos \theta \sin \theta & \sin ^{2} \theta-\sigma \cos ^{2} \theta
\end{array}\right),
$$

with $\theta$ the angle between the tension $\vec{T}$ and the $y$ axis $[\vec{T}=$ $\left.T \cos (\pi / 2+\theta) \vec{e}_{x}+T \sin (\pi / 2+\theta) \vec{e}_{y}\right],{ }^{35}$ and $\sigma$ the Poisson ratio, which takes the graphite value of 0.165 , which we choose in the remainder. ${ }^{36}$ The conclusions made by Pereira et al. are that (i) the minimal strain required for opening a gap is about $23 \%$, that (ii) tension along the zigzag direction $(\theta=0, \pi / 3,2 \pi / 3)$ is optimal for the opening of a band gap, and that (iii) tension along the armchair direction $(\theta=\pi / 2,5 \pi / 6,11 \pi / 6)$ never results in a band gap. From 
Eq. (28) it follows that the interplane coupling $t_{\perp}$ does not play any role and that the same conclusions are valid for the bilayer.

\section{CONCLUSIONS}

Applying strain to a graphene bilayer alters its electronic structure. Using the simplest tight-binding model, featuring only nearest-neighbor intra- and interplane hopping ( $t$ and $t_{\perp}$ ), we have shown that for the $A B$ (Bernal) stacking of two graphene sheets, the opening of a band gap by pushing (pulling) the two graphene layers toward (away from) each other is possible. As a consequence of the different neighborhoods experienced by $A$ and $B$ carbon atoms, the change in on-site tight-binding energies results in a symmetry breaking which, for large enough strains, opens a band gap. We find that pulling and pushing are inequivalent; the former is more effective in producing a gap. Predictions of the minimally required strain to open a band gap critically depend on values for the changes in on-site and interlayer hopping energies with interplane distance $\left(\frac{\partial V}{\partial c}\right.$ and $\left.\frac{\partial t_{\perp}}{\partial c}\right)$. These quantities are not readily available from the literature. We therefore rely on rules of thumb to make acceptable estimates, and find that for pulling, a band gap opens from $\varepsilon_{z z}=0.25$ onward. For $\varepsilon_{z z} \approx 30 \%$, the band gap is about $125 \mathrm{meV}$. For pushing, the critical strain is well beyond $50 \%$. Note that a strain of $50 \%$ would require a pressure of $p=0.5 c_{44} \approx 2 \mathrm{GPa}$ [taking for the elastic constant $c_{44}$ the value of graphite, $c_{44}=4.18 \mathrm{GPa}$ (Ref. 37)], which is experimentally feasible. While bringing the two layers close together can be realized by applying high pressure, pulling the graphene sheets away from each other is an experimental challenge. A possible indirect way to do so would be to intercalate the bilayer; recently, for example, $\mathrm{Li}$ atoms have successfully been intercalated between two graphene sheets. ${ }^{38}$ Interestingly, we find that at the critical strain, the electronic dispersion near the $K$ point consists of a triple degeneracy of two crossing linear bands (Dirac cone) and one parabolic band. This result shows that under certain symmetry-breaking strain conditions, bilayer graphene can exhibit Dirac fermions. Normally, Dirac fermions only occur in graphene multilayers consisting of an odd number of graphene sheets. ${ }^{39}$ The asymmetry of the hamiltonian matrix diagonal lies at the basis of the band-structure modifications observed in Fig. 2. It was already recognized before ${ }^{30}$ that intralayer on-site energy differences can lead to band gaps of a different type than the Mexican-hat-like band-gap band structures ${ }^{15-19}$ associated with interlayer energy differences, e.g., induced by the application of a bias. In the present work, we have shown that uniform perpendicular strain provides a physical means for enhancing the intralayer energy differences.

We also considered the possibility to push/pull parallel to the bilayer (symmetric uniform strain). It turns out that the criterion for opening a band gap is the same as for monolayer graphene; the interplane coupling $t_{\perp}$ does not play any role. The conclusions made by Pereira et al. ${ }^{21}$ for the monolayer can be transferred to the bilayer; a strain along the zigzag direction larger than $\sim 23 \%$ results in a band gap, while pushing/pulling along the armchair direction never leads to a gap.

Although having provided estimates for strains $\varepsilon$ required for the opening of a band gap and associated band-gap magnitudes $\Delta E_{\mathrm{g}}$, for the case of pushing/pulling perpendic- ularly, we wish to emphasize the qualitative aspect of our results. Irrespective of the precise values of the tight-binding parameters and derived quantities, it is the particular structure of the graphene bilayer that, when deformed, allows for gapped electronic structures. In our opinion, the deformation scenario shown here in which a band gap can be induced in bilayer graphene by perpendicular compression or expansion should stimulate experimental investigations on the possibility of strain-engineering bilayer graphene's electronic properties. Recalling graphene's high strength, this should be feasible. In addition, we suggest that the theoretical models presented here be reconsidered by means of precise ab initio calculations.

Note added in proof. A recent publication by Frank et al. ${ }^{40}$ reports on a Raman spectroscopy investigation of strained bilayer graphene. By means of a cantilever beam assembly, Bernal stacked bilayer graphene embedded in a polymer matrix was subjected to uniaxial stress. Raman spectroscopy was then used to map the resulting strain field. These experiments demonstrate a promising route for applying and characterising strain in bilayer graphene, and therefore provide an important additional experimental motivation for our theoretical work.

\section{ACKNOWLEDGMENTS}

The authors would like to acknowledge O. Leenaerts, E. Mariani, K. H. Michel, and J. Schelter for useful discussions. B.V. was financially supported by the Flemish Science Foundation (FWO-Vl). This work was financially supported by the ESF program EuroGraphene under projects CONGRAN and ENTS as well as by the DFG.

\section{APPENDIX: STRAIN}

As described in the main text, deformations of the bilayer graphene carbon network enter the tight-binding formalism via bond length changes [Eqs. (8a)-(9b)]. In this Appendix, we elaborate the expressions for $\delta a_{l}$ and $\delta c$ and the strained graphene bilayer tight-binding hamiltonian.

\section{Continuous description: Long-wavelength limit}

In general, one has

$$
\begin{aligned}
a & +\delta a_{l} \\
& =\left|\vec{X}+\vec{d}_{l}+\vec{u}\left(\vec{X}+\vec{d}_{l}\right)-[\vec{X}+\vec{u}(\vec{X})]\right| \\
& =\sqrt{a^{2}+2 \vec{d}_{l} \cdot\left[\vec{u}\left(\vec{X}+\vec{d}_{l}\right)-\vec{u}(\vec{X})\right]+\left|\vec{u}\left(\vec{X}+\vec{d}_{l}\right)-\vec{u}(\vec{X})\right|^{2}} .
\end{aligned}
$$

Assuming small bond length changes $\delta a_{l}$, we retain only firstorder terms:

$$
a+\delta a_{l} \approx a\left(1+\frac{1}{a^{2}} \vec{d}_{l} \cdot\left[\vec{u}\left(\vec{X}+\vec{d}_{l}\right)-\vec{u}(\vec{X})\right]\right),
$$

so that

$$
\delta a_{l} \approx \frac{1}{a} \vec{d}_{l} \cdot\left[\vec{u}\left(\vec{X}+\vec{d}_{l}\right)-\vec{u}(\vec{X})\right] .
$$

In the case of small bond length changes, the differences between displacements at neighboring sites $\left|\vec{u}\left(\vec{X}+\vec{d}_{l}\right)-\vec{u}(\vec{X})\right|$ must be small as well. In other words, the displacement field $\vec{u}$ is a slowly varying (vector) function of $\vec{X}$, implying that a 
linearization of $\vec{u}$ at $\vec{X}$ approximates $\vec{u}$ at $\vec{X}+\vec{d}_{l}$ well enough. In this so-called long-wavelength limit we then have

$$
\left.\vec{d}_{l} \cdot\left[\vec{u}\left(\vec{X}+\vec{d}_{l}\right)-\vec{u}(\vec{X})\right] \approx\left(\vec{d}_{l} \cdot \vec{\nabla}\right)\left(\vec{d}_{l} \cdot \vec{u}\right)\right|_{\vec{X}} .
$$

The operator $\vec{\nabla}=\left(\frac{\partial}{\partial x}, \frac{\partial}{\partial y}, \frac{\partial}{\partial z}\right)$ acts on $\vec{u}(\vec{X})$ with $\vec{X}$ taken as the continuous spatial variable $\vec{x}=(x, y, z)$. Writing $d_{l \alpha}, \alpha=1,2$ for the $x$ and $y$ components of $\vec{d}_{l}\left(\vec{d}_{l}\right.$ has no $z$ component; see top of Fig. 1), we obtain

$$
\left.\left(\vec{d}_{l} \cdot \vec{\nabla}\right)\left(\vec{d}_{l} \cdot \vec{u}\right)\right|_{\vec{X}}=\left.\sum_{\alpha=1}^{2} \sum_{\beta=1}^{2} d_{l \alpha} d_{l \beta} \frac{\partial u_{\beta}}{\partial x_{\alpha}}\right|_{\vec{X}} .
$$

In the following, we drop the $\left.\right|_{\vec{X}}$ attributes and keep in mind that there formally is a dependence on the position in the lattice. The expression for $\delta a_{l}$ now becomes

$$
\delta a_{l} \approx \frac{1}{a} \sum_{\alpha=1}^{2} \sum_{\beta=1}^{2} d_{l \alpha} d_{l \beta} \frac{\partial u_{\beta}}{\partial x_{\alpha}} .
$$

Calculating explicit expressions for the quantities $D_{\alpha \beta}^{l}=$ $d_{l \alpha} d_{l \beta}$ leads to

$$
\begin{aligned}
D^{1} & =\frac{a^{2}}{4}\left(\begin{array}{cc}
1 & \sqrt{3} \\
\sqrt{3} & 3
\end{array}\right), \quad D^{2}=\frac{a^{2}}{4}\left(\begin{array}{cc}
1 & -\sqrt{3} \\
-\sqrt{3} & 3
\end{array}\right), \\
D^{3} & =a^{2}\left(\begin{array}{ll}
1 & 0 \\
0 & 0
\end{array}\right) .
\end{aligned}
$$

In a completely analogous way we obtain for $\delta c$-recalling that $\vec{c}=c \vec{e}_{z}$ - the following expression (up to first order):

$$
\delta c=|\vec{X}+\vec{c}+\vec{u}(\vec{X}+\vec{c})-[\vec{X}+\vec{u}(\vec{X})]|-c \approx c \frac{\partial u_{z}}{\partial z} .
$$

\section{Corrections to the hamiltonian matrix}

The corrections to the hamiltonian involve the following quantities:

$$
\begin{gathered}
\delta U_{\mathrm{A}}=\sum_{l=1}^{3} \frac{\partial V}{\partial a} \delta a_{l}+\frac{\partial V}{\partial c} \delta c=\frac{3 a}{2} \frac{\partial V}{\partial a}\left(\varepsilon_{x x}+\varepsilon_{y y}\right)+c \frac{\partial V}{\partial c} \varepsilon_{z z}, \\
\delta U_{\mathrm{B}}=\sum_{l=1}^{3} \frac{\partial V}{\partial a} \delta a_{l}=\frac{3 a}{2} \frac{\partial V}{\partial a}\left(\varepsilon_{x x}+\varepsilon_{y y}\right) .
\end{gathered}
$$

Here, use has been made of the identity

$$
\sum_{l=1}^{3} D^{l}=\frac{3 a^{2}}{2}\left(\begin{array}{ll}
1 & 0 \\
0 & 1
\end{array}\right)
$$

and the definition of the (first-order) components of the strain tensor:

$$
\varepsilon_{i j}=\frac{1}{2}\left(\frac{\partial u_{i}}{\partial x_{j}}+\frac{\partial u_{j}}{\partial x_{i}}\right)
$$

Note that the strain tensor is, in general, space dependent: $[\varepsilon] \equiv[\varepsilon](\vec{X})$. Therefore transforming the summation

$\sum_{\vec{X}_{1}} c_{\vec{X}_{1}}^{\dagger} \delta U^{\mathrm{A}} c_{\vec{X}_{1}}=\sum_{\vec{X}_{1}} c_{\vec{X}_{1}}^{\dagger}\left[\frac{3 a}{2} \frac{\partial U}{\partial a}\left(\varepsilon_{x x}+\varepsilon_{y y}\right)+c \frac{\partial V}{\partial c} \varepsilon_{z z}\right] c_{\vec{X}_{1}}$

into a summation over $\vec{q}$ vectors involves discrete Fourier transforms:

$$
\begin{aligned}
\sum_{\vec{X}_{1}} c_{\vec{X}_{1}}^{\dagger} \delta U^{\mathrm{A}} c_{\vec{X}_{1}}= & \frac{1}{\sqrt{N}} \sum_{\vec{q}} \sum_{\vec{q}^{\prime}} c_{\mathrm{A}_{1}}^{\dagger}(\vec{q})\left[\frac { 3 a } { 2 } \frac { \partial U } { \partial a } \left[\varepsilon_{x x}\left(\vec{q}^{\prime}-\vec{q}\right)\right.\right. \\
& \left.\left.+\varepsilon_{y y}\left(\vec{q}^{\prime}-\vec{q}\right)\right]+c \frac{\partial V}{\partial c} \varepsilon_{z z}\left(\vec{q}^{\prime}-\vec{q}\right)\right] c_{\mathrm{A}_{1}}\left(\vec{q}^{\prime}\right), \\
\varepsilon_{i j}(\vec{q})= & \frac{1}{\sqrt{N}} \sum_{\vec{X}_{i}} \varepsilon_{i j}\left(\vec{X}_{i}\right) e^{i \vec{q} \cdot \vec{X}_{i}} .
\end{aligned}
$$

Importantly, when the strain tensor $[\varepsilon]$ is space independent, i.e., in the case of uniform deformations, Eqs. (A13a) and (A13b) collapse into

$$
\begin{aligned}
& \sum_{\vec{X}_{1}} c_{\vec{X}_{1}}^{\dagger} \delta U^{\mathrm{A}} c_{\vec{X}_{1}} \\
& \quad=\sum_{\vec{q}} c_{\mathrm{A}_{1}}^{\dagger}(\vec{q})\left[\frac{3 a}{2} \frac{\partial U}{\partial a}\left(\varepsilon_{x x}+\varepsilon_{y y}\right)+c \frac{\partial U}{\partial c} \varepsilon_{z z}\right] c_{\mathrm{A}_{1}}(\vec{q})
\end{aligned}
$$

so that

$$
\delta \mathfrak{H}_{11}=\frac{3 a}{2} \frac{\partial U}{\partial a}\left(\varepsilon_{x x}+\varepsilon_{y y}\right)+c \frac{\partial U}{\partial c} \varepsilon_{z z}
$$

is $\vec{q}$ independent. For the correction to $\mathfrak{H}_{22}$ we then have

$$
\delta \mathfrak{H}_{22}=\frac{3 a}{2} \frac{\partial U}{\partial a}\left(\varepsilon_{x x}+\varepsilon_{y y}\right) .
$$

The off-diagonal nonzero hopping matrix elements are more complicated. We will need the quantities

$$
\begin{aligned}
\delta t_{l} & =\frac{\partial t}{\partial a} \delta a_{l}=\frac{1}{a} \frac{\partial t}{\partial a} \sum_{\alpha=1}^{2} \sum_{\beta=1}^{2} D_{\alpha \beta}^{l} \frac{\partial u_{\beta}}{\partial x_{\alpha}}, \\
\delta t_{\perp} & =\frac{\partial t_{\perp}}{\partial c} \delta c=c \frac{\partial t_{\perp}}{\partial c} \frac{\partial u_{z}}{\partial z} .
\end{aligned}
$$

Let us consider the term

$$
\begin{aligned}
-\sum_{\vec{X}_{1}} \sum_{l=1}^{3} c_{\vec{X}_{1}}^{\dagger} \delta t_{l} c_{\vec{X}_{1}+\vec{d}_{l}}= & -\frac{1}{\sqrt{N}} \frac{1}{a} \frac{\partial t}{\partial a} \sum_{\vec{q}} \sum_{\vec{q}^{\prime}} \sum_{\alpha=1}^{2} \sum_{\beta=1}^{2} c_{\mathrm{A}_{1}}^{\dagger} \\
& \times(\vec{q}) e_{\alpha \beta}\left(\vec{q}^{\prime}-\vec{q}\right) F_{\alpha \beta}\left(\vec{q}^{\prime}\right) c_{\mathrm{B}_{1}}\left(\vec{q}^{\prime}\right),
\end{aligned}
$$

with

$$
\begin{aligned}
& F_{\alpha \beta}(\vec{q})=\sum_{l=1}^{3} D_{\alpha \beta}^{l} e^{i \vec{q} \cdot \vec{d}_{l}}, \\
& e_{\alpha \beta}(\vec{q})=\frac{1}{\sqrt{N}} \sum_{\vec{X}_{1}} e^{i \vec{q} \cdot \vec{X}_{1}} \frac{\partial u_{\beta}}{\partial x_{\alpha}} .
\end{aligned}
$$


Again, in the case of uniform deformations, $\frac{\partial u_{\beta}}{\partial x_{\alpha}}$ is space independent and Eq. (A18) simplifies to

$$
-\sum_{\vec{X}_{1}} \sum_{l=1}^{3} c_{\vec{X}_{1}}^{\dagger} \delta t_{l} c_{\vec{X}_{1}+\vec{d}_{l}}=-\frac{1}{a} \frac{\partial t}{\partial a} \sum_{\vec{q}} c_{\mathrm{A}_{1}}^{\dagger}(\vec{q}) \sum_{\alpha=1}^{2} \sum_{\beta=1}^{2} F_{\alpha \beta}(\vec{q}) \frac{\partial u_{\beta}}{\partial x_{\alpha}} c_{\mathrm{B}_{1}}(\vec{q}),
$$

so that the matrix element $\delta \mathfrak{H}_{12}$ becomes

$$
\delta \mathfrak{H}_{12}=-\frac{1}{a} \frac{\partial t}{\partial a} \sum_{\alpha=1}^{2} \sum_{\beta=1}^{2} F_{\alpha \beta}(\vec{q}) \frac{\partial u_{\beta}}{\partial x_{\alpha}}
$$

For $F(\vec{q})$ one obtains

$$
\begin{aligned}
F(\vec{q}) & =a^{2}\left(\begin{array}{cc}
\frac{1}{4}\left(e^{i \vec{q} \cdot \vec{d}_{1}}+e^{i \vec{q} \cdot \vec{d}_{2}}\right)+e^{i \vec{q} \cdot \vec{d}_{3}} & \frac{\sqrt{3}}{4}\left(e^{i \vec{q} \cdot \vec{d}_{1}}-e^{i \vec{q} \cdot \vec{d}_{2}}\right) \\
\frac{\sqrt{3}}{4}\left(e^{i \vec{q} \cdot \vec{d}_{1}}-e^{i \vec{q} \cdot \vec{d}_{2}}\right) & \frac{3}{4}\left(e^{i \vec{q} \cdot \vec{d}_{1}}+e^{i \vec{q} \cdot \vec{d}_{2}}\right)
\end{array}\right) \\
& =a^{2} e^{i(1 / 2) a q_{x}}\left(\begin{array}{cc}
\frac{1}{2} \cos \left(\frac{\sqrt{3}}{2} a q_{y}\right)+e^{-i(3 / 2) a q_{x}} & \frac{\sqrt{3}}{2} i \sin \left(\frac{\sqrt{3}}{2} a q_{y}\right) \\
\frac{\sqrt{3}}{2} i \sin \left(\frac{\sqrt{3}}{2} a q_{y}\right) & \frac{3}{2} \cos \left(\frac{\sqrt{3}}{2} a q_{y}\right)
\end{array}\right) .
\end{aligned}
$$

Near the $K$ point, one has

$$
F_{\alpha \beta}\left(\vec{q}_{\mathrm{K}}+\vec{k}\right)=\frac{3 a^{2}}{4} e^{i(\pi / 3)}\left(\begin{array}{cc}
-1+\frac{a}{2}\left(3 i k_{x}-k_{y}\right) & i-\frac{a}{2}\left(k_{x}-i k_{y}\right) \\
i-\frac{a}{2}\left(k_{x}-i k_{y}\right) & 1+\frac{a}{2}\left(i k_{x}-3 k_{y}\right)
\end{array}\right) .
$$

The phase factor $e^{i(\pi / 3)}$ can be eliminated by redefining the creation and annihilation operators for electrons at $B$ sites (position vectors $\vec{X}_{1}+\vec{d}_{l}$ and $\left.\vec{X}_{2}-\vec{d}_{l}\right)$ :

$$
\begin{gathered}
c_{\vec{X}_{i} \pm \vec{d}_{l}}^{\dagger} \longrightarrow c_{\vec{X}_{i} \pm \vec{d}_{l}}^{\dagger} e^{-i(\pi / 6)} \\
c_{\vec{X}_{i} \pm \vec{d}_{l}} \longrightarrow c_{\vec{X}_{i} \pm \vec{d}_{l}} e^{i(\pi / 6)}
\end{gathered}
$$

Near the $K$ point, we obtain the following correction to the matrix element:

$$
\delta \mathfrak{H}_{12}(\vec{k})=-i \frac{\partial t}{\partial a} \frac{3 a}{4}\left[\varepsilon_{x x}\left(-1+\frac{a}{2}\left(3 i k_{x}-k_{y}\right)\right)+\varepsilon_{y y}\left(1+\frac{a}{2}\left(i k_{x}-3 k_{y}\right)\right)+2 \varepsilon_{x y}\left(i-\frac{a}{2}\left(k_{x}-i k_{y}\right)\right)\right],
$$

where the factor $i$ comes from multiplying the phase factors $e^{i(\pi / 3)}$ and $e^{i(\pi / 6)}$, and where the definition of the strain tensor in Eq. (A11) has been used. For small deformations, the terms proportional to $\varepsilon_{\alpha \beta} k$, with $\alpha, \beta \in\{x, y\}$, can be neglected:

$$
\delta \mathfrak{H}_{12}^{\mathrm{K}}=-i \frac{\partial t}{\partial a} \frac{3 a}{4}\left(-\varepsilon_{x x}+\varepsilon_{y y}+2 i \varepsilon_{x y}\right) .
$$

We now consider the term

$$
\begin{gathered}
\sum_{\vec{X}_{1}} \delta t_{\perp} c_{\vec{X}_{1}}^{\dagger} c_{\vec{X}_{1}+\vec{c}}=\frac{1}{\sqrt{N}} c \frac{\partial t_{\perp}}{\partial c} \sum_{\vec{q}} \sum_{\vec{q}^{\prime}} c_{\mathrm{A}_{1}}^{\dagger}(\vec{q}) f\left(\vec{q}^{\prime}-\vec{q}\right) e^{i \vec{q}^{\prime} \cdot \vec{c}} c_{\mathrm{A}_{2}}\left(\vec{q}^{\prime}\right), \\
f(\vec{q})=\frac{1}{\sqrt{N}} \sum_{\vec{X}_{1}} e^{i \vec{q} \cdot \vec{X}_{1}} \frac{\partial u_{z}}{\partial z} .
\end{gathered}
$$

Here, the factor $e^{i \vec{q}^{\prime} \cdot \vec{c}}$ is equal to 1 because $\vec{q}^{\prime}$ (only $x$ and $y$ components) and $\vec{c}$ (only $z$ component) are perpendicular. For space-independent $\frac{\partial u_{z}}{\partial z}$ we obtain

$$
\sum_{\vec{X}_{1}} \delta t_{\perp} c_{\vec{X}_{1}}^{\dagger} c_{\vec{X}_{1}+\vec{c}}=c \frac{\partial t_{\perp}}{\partial c} \frac{\partial u_{z}}{\partial z} \sum_{\vec{q}} c_{\mathrm{A}_{1}}^{\dagger}(\vec{q}) c_{\mathrm{A}_{2}}(\vec{q})=c \frac{\partial t_{\perp}}{\partial c} \varepsilon_{z z} \sum_{\vec{q}} c_{\mathrm{A}_{1}}^{\dagger}(\vec{q}) c_{\mathrm{A}_{2}}(\vec{q}),
$$

so that the correction to the matrix element $\mathfrak{H}_{13}$ reads

$$
\delta \mathfrak{H}_{13}=c \frac{\partial t_{\perp}}{\partial c} \varepsilon_{z z} .
$$

These considerations then lead to the correction $\delta \mathfrak{H}$ to the hamiltonian matrix $\mathfrak{H}$ given by Eqs. (10) and (11). 
${ }^{1}$ K. S. Novoselov, A. K. Geim, S. V. Morosov, D. Jiang, Y. Zhang, S. V. Dubonos, I. V. Griorieva, and A. A. Firsov, Science 306, 666 (2004).

${ }^{2}$ A. K. Geim and K. S. Novoselov, Nat. Mater. 6, 183 (2007).

${ }^{3}$ C. W. J. Beenakker, Rev. Mod. Phys. 80, 1337 (2008).

${ }^{4}$ A. K. Geim, Science 324, 1530 (2009).

${ }^{5}$ A. H. Castro Neto, F. Guinea, N. M. R. Peres, K. S. Novoselov, and A. K. Geim, Rev. Mod. Phys. 81, 109 (2009).

${ }^{6}$ S. Das Sarma, S. Adam, E. H. Hwang, and E. Rossi, Rev. Mod. Phys. 83, 407 (2011).

${ }^{7}$ K. S. Novoselov, Rev. Mod. Phys. 83, 837 (2011).

${ }^{8}$ A. K. Geim, Rev. Mod. Phys. 83, 851 (2011).

${ }^{9}$ K. S. Novoselov, D. Jiang, F. Schedin, T. J. Booth, V. V. Khotkevich, S. V. Morosov, and A. K. Geim, Proc. Natl. Acad. Sci. USA 102, 10451 (2005).

${ }^{10}$ K. S. Novoselov, A. K. Geim, S. V. Morozov, D. Jiang, M. I. Katsnelson, I. V. Griorieva, S. V. Dubonos, and A. A. Firsov, Nature (London) 438, 197 (2005).

${ }^{11}$ Y. Zhang, Y.-W. Tan, H. L. Stormer, and P. Kim, Nature (London) 438, 201 (2005).

${ }^{12}$ M. Koshino and T. Ando, Phys. Rev. B 73, 245403 (2006).

${ }^{13}$ J. Nilsson, A. H. Castro Neto, N. M. R. Peres, and F. Guinea, Phys. Rev. B 73, 214418 (2006).

${ }^{14}$ B. Partoens and F. M. Peeters, Phys. Rev. B 74, 075404 (2006).

${ }^{15}$ E. McCann and V. I. Fal'ko, Phys. Rev. Lett. 96, 086805 (2006).

${ }^{16}$ F. Guinea, A. H. Castro Neto, and N. M. R. Peres, Phys. Rev. B 73, 245426 (2006).

${ }^{17}$ T. Ohta, A. Bostwick, T. Seyller, K. Horn, and E. Rotenberg, Science 313, 951 (2006).

${ }^{18}$ E. McCann, Phys. Rev. B 74, 161403 (2006).

${ }^{19}$ E. V. Castro, K. S. Novoselov, S. V. Morozov, N. M. R. Peres, J. M. B. Lopes dos Santos, J. Nilsson, F. Guinea, A. K. Geim, and A. H. Castro Neto, Phys. Rev. Lett. 99, 216802 (2007).

${ }^{20}$ F. Guinea, M. I. Katsnelson, and A. K. Geim, Nat. Phys. 6, 30 (2010)
${ }^{21}$ V. M. Pereira, A. H. Castro Neto, and N. M. R. Peres, Phys. Rev. B 80, 045401 (2009).

${ }^{22}$ S. H. Lee, C. W. Chiu, Y. H. Ho, and M. F. Lin, Synth. Met. 160, 2435 (2010).

${ }^{23}$ E. Mariani, A. J. Pearce, and F. von Oppen, e-print arXiv:1110.2769 (to be published).

${ }^{24}$ M. Mucha-Kruczyński, I. L. Aleiner, and V. I. Fal'ko, Phys. Rev. B 84, 041404(R) (2011).

${ }^{25}$ M. Mucha-Kruczyński, E. McCann, and V. I. Fal'ko, Solid State Commun. 151, 1088 (2011).

${ }^{26}$ B. R. K. Nanda and S. Satpathy, Phys. Rev. B 80, 165430 (2009).

${ }^{27}$ H. Raza and E. C. Kan, J. Phys.: Condens. Matter 21, 102202 (2009).

${ }^{28}$ S.-M. Choi, S.-H. Jhi, and Y.-W. Son, Nano Lett. 10, 3486 (2010).

${ }^{29}$ D. D. L. Chung, J. Mater. Sci. 37, 1475 (2002).

${ }^{30}$ M. Mucha-Kruczyński, I. L. Aleiner, and V. I. Fal'ko, Semicond. Sci. Technol. 25, 033001 (2010).

${ }^{31}$ H. Suzuura and T. Ando, Phys. Rev. B 65, 235412 (2002).

${ }^{32}$ J. Bardeen and W. Shockley, Phys. Rev. 80, 70 (1950).

${ }^{33}$ W. A. Harrison, Elementary Electronic Structure (World Scientific, Singapore, 1999).

${ }^{34}$ S. Reich, J. Maultzsch, C. Thomsen, and P. Ordejón, Phys. Rev. B 66, 035412 (2002)

${ }^{35}$ The $x$ and $y$ axes of Pereira et al. (Ref. 21) are rotated over $\pi / 2$ with respect to the axes of the present work.

${ }^{36}$ L. Blakslee, D. G. Proctor, E. J. Seldin, G. B. Stence, and T. Wen, J. Appl. Phys. 41, 3373 (1970).

${ }^{37}$ K. H. Michel and B. Verberck, Phys. Rev. B 78, 085424 (2008).

${ }^{38}$ K. Sugawara, K. Kanetani, T. Sato, and T. Takahashi, AIP Advances 1, 022103 (2011).

${ }^{39}$ B. Partoens and F. M. Peeters, Phys. Rev. B 75, 193402 (2007).

${ }^{40}$ O. Frank, M. Bousa, I. Riaz, R. Jalil, K. S. Novoselov, G. Tsoukleri, J. Parthenios, L. Kavan, K. Papagelis, and C. Galiotis, Nano Lett. 12, 687 (2012). 